\title{
Organizational Transformation and Process Modeling in Modern Telecommunications Companies
}

\author{
D.M. Emiris, D.E. Koulouriotis, Z. Chourmouziadou, V. Moustakis, N. Bilalis \\ Department of Production Engineering and Management, Technical University of Crete \\ 73100 Chania, Crete
}

\begin{abstract}
The telecommunications industry has presented a healthy growth over the recent years, and generated combined revenues of approximately 700 billion Euros in 1995 worldwide, while it is estimated that in 2005, this number will rise to 970 billion Euros in the European Union alone. This growth is directly associated, apart of the strong direct dependence on informatics and digital technology, to the international policies followed, as regards the transition from a monopolistic environment towards a liberalized one, through various deregulation stages. Despite the obvious benefits for the end-user, which are further emphasized as a deregulation process evolves, the telecommunications market providers must face a continuously changing environment, which necessitates the research, selection and adoption of new technologies, the flexibility to adapt to rapid market changes through focused and flexible marketing programs, the agility to provide customized services, and the ability to integrate services, solutions, and equipment for the end-users. A critical step in the attempt to meet the above requirements is business modeling. The present work examines organizational methodologies for an entrant small-size telecom company, which would attempt to meet the above criteria. The study introduces the concept of process modeling in a general business environment and analyses the way in which a telecom company should be organized and structured. A SWOT (Strengths-Weaknesses-Opportunities-Threats) analysis initially defines the operational environment of the corporation. Case models are created as interim stages for the support of the interactions between the business entities. The external influences are enumerated and their role is highlighted. The business processes are then identified, analyzed and evaluated, and typical process flowcharts are generated and documented.
\end{abstract}

Key words: telecommunications, process modeling

The original version of this chapter was revised: The copyright line was incorrect. This has been corrected. The Erratum to this chapter is available at DOI: 10.1007/978-0-387-35492-7_50 


\section{INTRODUCTION}

The telecommunications industry has presented a healthy growth over the recent years, and generated combined revenues of approximately 700 billion Euros in 1995 worldwide, while it is estimated that in 2005, this number will rise to 970 billion Euros in the European Union alone. This growth is directly associated, apart of the strong direct dependence from informatics and digital technology, to the international policies followed, as regards the transition from a monopolistic environment towards a liberalized one, through various deregulation stages. The EU, in particular, maintains a balanced policy between deregulation and obedience to the competition rules, and is based on several axes of priority, such as, the generation of new employment positions, the encouragement of technological innovation, the provision of the ability to the citizens to profit of the information society benefits, and the creation of conditions which will enable European corporations to strengthen their position in the international market.

Despite the obvious benefits for the end-user, which are further emphasized as a deregulation process evolves, the telecommunications market is a most competitive one for the involved corporations, not only the equipment manufacturers and technology generators, but also for the service provision ones. The latter face a continuously changing environment, which necessitates the research, selection and adoption of new technologies, the flexibility to adapt to rapid market changes through focused and flexible marketing programs, the agility to provide customized services, and the ability to integrate services, solutions, and equipment for the end-users. These factors often require the change of the operating philosophy for the incumbent telecom corporations, and certainly require a modern and flexible development strategy for new ones.

A critical step in the attempt to meet the above requirements is business modeling. This term is used herein to describe a symbolic language or protocol, both for representation and communication purposes, which includes graphical means (e.g. diagrams, flowcharts, other visual references) to depict inter-relationships and supporting means (e.g. reports). The need for business modeling stems from the need for effective knowledge management, efficient information flow within a company, support of basic processes and operations, and understanding the current conditions so as to enable future improvements [1,3]. There occur, however, certain conflicting concerns as regards the business modeling, namely: (a) the fact that the more complex the structure of a corporation is, the most complex, and thus more inflexible to changes, will be the business models that describe it; and (b) the fact that business models need to be quite detailed in order to be efficient 
and accurate, thus making them inefficient for information exchange and knowledge management.

The present work examines organizational methodologies for an entrant small-size telecom company, which would attempt to meet the above criteria. The study introduces the concept of process modeling in a general business environment and analyses the way in which a telecom company should be organized and structured. The common denominator in this attempt is the need to increase the productivity within a lean enterprise. The term lean is used to describe the ability of a corporation to produce added value at a low cost, so that the end-user receives an advanced service at a proper price. In this study, it is shown that the process-oriented management of a corporation enables to achieve a lean operation. Typical management schemes are contrasted and compared. The cost for the transition from an existing structured to a process-oriented structure, is investigated and identified.

The study applies the theoretical approaches to an actual telecom

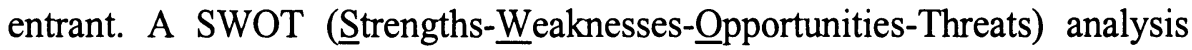
initially defines the operational environment of the corporation. Case models are created as interim stages for the support of the interactions between the business entities. The external influences are enumerated and their role is highlighted. The business processes are then identified, analyzed and evaluated, and typical process flowcharts are generated and documented.

Several interesting conclusions result from this work. First, it is shown that for the successful representation of a company's operation, several software tools for process modelling and documentation are needed. It is recognized that, especially in the telecom market, today's corporations need to adopt a more flexible, process-oriented business structure. The analysis and modelling of business processes facilitates the identification and assignment of roles in an organization chart and enables a company to provide customized, client-oriented services through efficient processes, thus remaining effective and profitable in a competitive, rapidly-changing, disintermediative, digital techno-economic environment.

\section{THE NEED FOR BUSINESS MODELING}

The need to understand thoroughly and manage effectively the information in a company, has led to methods and techniques for the systematic organization of knowledge. A most important organization tool for knowledge and information is modeling. Modeling permits the discretization of knowledge and its presentation in a commonly perceivable 
manner, focusing in the importance of the human factor, as a company is inherently defined by the interactions between humans. In an attempt to identify the need for modeling in a company, it is important to stress the definition for a company. The nature of a company may be easily understood under the prism of relationships. A company (or an operation) may thus be defined as a closed network of interrelated persons, and is characterized by a unique identity. These relationships are responsible for the creation of the unique company identity and provide a coherence and integrity sense [2].

The identity of an operation, however, does not depend from the eventual persons: as long as the persons involved satisfy the given interrelationship system, they can be any. On the other hand, if these relationships change, even if the people remain the same, the operation will also change; it will create a new identity. The identity of the company is directly related to its goal and its field of operation, thus permitting several companies to have similar identities. However, what makes a company unique is its structure, that is the way that the inter-relationships, which are developed within the company's frame, are shaped. In other words, it is the way a company is organized so as to achieve its goals. The need to understand in depth a company is thus translated to the need to depict and understand the inter-relationship system, from different perspective angles and different degrees of depth. Such an approach may be achieved through the modeling of information and the production of respective description reports $[4,5,6]$.

The main obstacle in the attempt to deepen in a company's operation is the analysis and understanding of the interactions within the company. The use of a verbal code of communication in such an approach introduces a significant degree of fuzziness and cannot be considered efficient. On the other hand, the use of a specific terminology, although it may facilitate a department or a special team, by no means can meet the needs of the entire company and thus to be used for knowledge transfer. As a result, a technique for structuring a written code of communication in a way as to avoid fuzziness and to result in perceivable depiction forms is needed. In this context, business modeling is a widely used, efficient method to deepen into and to transfer information in a business environment.

Several reasons may trigger the modeling of a company's systemic components. The need to organize and manage systematically the information and knowledge (either provided or acquired) within a company, apart of being the main target of the knowledge management field, is the first reason, as it uses broadly modeling tools and emphasizes on the human factor. Secondly, the development of models within an operation may be 
used as a means for documentation and for personnel training in complex systems. The use of extensive documents, where processes and functions of an operation are described, is not the most recommended tools of knowledge transfer, as the length of a document may be an inhibiting factor in gathering the personnel's attention, as required. In contrary, the use of a diagram with a short descriptive report is much more likely to draw the attention. Since each person categorizes its duties with respect to the time that these require, by prioritizing them in an increasing order of time required, it is more probable that someone deals with a model rather than with an extensive document. Apart of this reason, the timesavings themselves are an important motive for development of business models, in order to communicate knowledge and information within a company.

Business modeling may also be triggered by the need to document the basic functions and processes that a company executes, as a risk prevention measure to protect the company from key people's leaving, moving, or substitution. Such a need arises each time a company's organizational structure undergoes a significant change, as in the cases, for example, of new product development, expansion to new markets, mergers, acquisitions, or buy-outs. In any respective case, the documentation of the basic processes and functions may offer a standardization pattern for the mode of operation and to create a basis for adaptation to changes, modifications or integration of new processes. Even in the case that a change in the organizational structure does not take place the processes documentation may be activated by the need to standardize the processes of product development and service provision.

Finally, the need to perform changes for the improvement of performance in a company may also trigger attempts to model the organization structure and the developed inter-relationships. Business modeling serves as a useful technique to conceive the present situation within a company and the faster examination of alternative improvement propositions. Through a model, several problematic points may be revealed, along with deficiencies and insufficiencies of the existing system. At the same time, the cost for the simulation of a system and for the examination of potential consequences caused by changes is much smaller compared to the recovery cost of a company after an unsuccessful deployment of improvement plans. The development of business models is thus encouraged before any attempt for re-organization or review within a company.

The development of business models is a fairly complicate task and it requires dexterities that should be developed. At the same time, there result three basic dilemmas as to the graphical representation of information, the nature of which needs to be understood, so as to efficiently deal with 
them. These dilemmas are further related to the diversification of the models developed. These dilemmas are:

- The complexity dilemma: The more complex is the structure of an operation, the more complicate become the models that depict it. As a result, their margins of flexibility in description become narrower. The answer to this dilemma is that the models should be kept as simple as possible. Any information that they carry must serve a purpose otherwise it should be omitted along with self-explanatory elements.

- The control models dilemma: The more complex a model is, the more difficult is for its inherent information to be communicated and perceived; however, control cannot be made efficient if simple models are used. The answer to this dilemma is that, on the one hand models should be kept as simple as possible by integrating only absolutely necessary information, but on the other hand, there should be a development within a company of such conditions as to permit the people who deal with monitoring and control to understand more complicate models. This may be achieved through the use of a common modeling code and continuous training.

- The communication dilemma: The transmission of knowledge and information within a company requires communication models rather than complex control models or models of composite information systems; otherwise, communication is not successful. The answer to this dilemma is that each model should be oriented to its user; that is, that a different model should be created for every possible user. In any case, a model is created in order to serve a purpose therefore, its role is predetermined. This role defines the complexity that each model should have, as well as the balance it should maintain.

\section{COMPANY DESCRIPTION}

The present work has examined organizational methodologies for an entrant small-size telecom company in an attempt to increase the productivity within a lean enterprise. The term lean is used to describe the ability of a corporation to produce added value at a low cost, so that the enduser receives an advanced service at a proper price. The steps outlined below have been followed in order to fulfill this task: 
- The analytical organization plan has been developed and scrutinized, and the basic interfaces between the company's departments have been identified.

- The models for each business unit have been shaped along with the interactions among them, and between them and the external environment.

- The feasibility analysis, which in essence moves a company, has been implemented and the required entities that interact with the operation have been identified, as well as the job descriptions and their inter-relationships.

- The SWOT (Strengths-Weaknesses-Opportunities-Threats) analysis has been performed (Figure 1).

\section{Strengths}

- High quality technical infrastructure that enables processing of a large volume of simultaneous calls

- Experienced personnel in telecom networks and troubleshooting

- Call routing alternatives

\section{Opportunities}

- Ability to provide data services

- Telecommunication network expansion and new markets deployment

- Search for competitive prices for call termination

- Ability to provide services at better rates than our competitors

- Ability to provide value-added services

\section{Weaknesses}

- Absence of integrated marketing policy

- Human resource responsibilities happen to overlap or generate gaps

\section{Threats}

- Fail to provide integrated telecommunication services

- Competitive pricing policy

- Loss of market share due to competitor's aggressive marketing strategy

Figure 1: SWOT Analysis.

The analysis of interactions, in any company, follows the principle that, for every department and process, one needs to produce outputs (e.g. products, services, etc.) using appropriate resources. The production of outputs requires the consumption or modification of input material, 
following certain rules or policies. As a result, assuming that in every operation and every department there exist certain processes executed, one can identify the above characteristics by studying the role of each process. These characteristics are: (i) the inputs (material, or information consumed or modified through the process), (ii) the outputs (materials, products, or information produced by the process), (iii) mechanisms and resources (key people, equipment, machinery, systems, etc.), and, (iv) controls (rules, policies, processes, limitations, orders, standards, etc., that define the process. These are depicted in Figure 2. Their fundamental property is that each characteristic may be further decomposed to a lower level of analysis, and vice versa, that is, each arrow in a specific level of analysis may result from the synthesis of partial characteristics of lower levels of analysis.

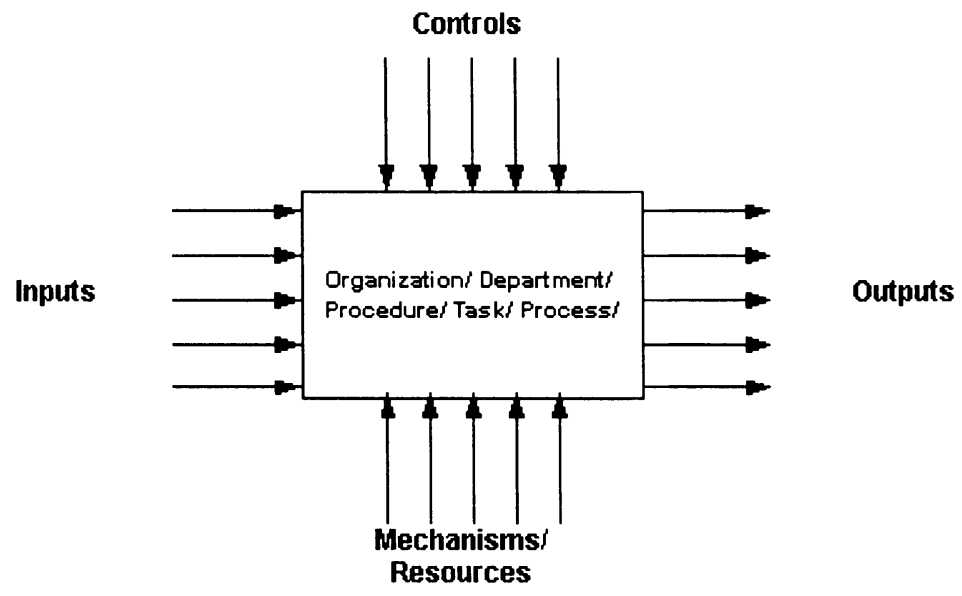

Figure 2: Characteristics of Processes.

The first step in the analysis of interactions includes the specification of the above characteristics for each process at every level, starting from the company as a whole and proceeding to the most detailed level. Such specification typically starts from the outputs, which are simpler to determine. Every process serves a specific purpose, which produces certain products. In the case where these products are not clearly specified or known, or they cannot be determined, then the process itself becomes questionable in terms of its need for existence. The second step is to determine the input data or material, which are consumed or transformed within the process context, so as to produce the outputs. Although rare, there exists the possibility that the input data and the output products are coincident; this would mean that the process does not produce an added value, and should be examined in terms of its need for existence. The difference between input data and output products may be specified 
by appropriate adjectives, which characterize the transformation that the process executes.

Once the input data are determined, the next step is to identify the mechanisms or resources, which are used for the process integration. The final step is the identification of the control under which a process is executed. All processes should be executed under at least one type of control. In cases where the identification of certain characteristics, such as inputs or controls, is fuzzy, then it is preferable for these to be characterized as controls as they constitute a form of input for every process.

The above type of analysis is depicted in Figures 3 and 4. In the first one, the case models (or main processes models) are shown along with their interrelationships for the company under study. Case models are interim models generated for the purpose of depicting cases and the way that business processes support certain interactions with external factors. In the second figure, one such main process, namely, the "New Customer Entry" process is further analyzed at a lower level, and the component processes are depicted along with their interactions and the interfaces with the outer environment.

Once the case models are created, they need to be decoded. Despite the fact that there is no established decoding method, in principle, inputs from the external environment are considered as data inputs or process activation commands. Similarly, outputs from the company to the external environment are considered as products or services generated from certain processes. Finally, the relationships between models signify interim products or services for inner customers or outer suppliers, or activation items for certain processes, such as the supply management when the supply control demonstrates insufficiency of the needs. For each case model, there may result one or more processes, which may be analyzed in turn, in subsequent levels of decomposition until simple tasks are determined. 


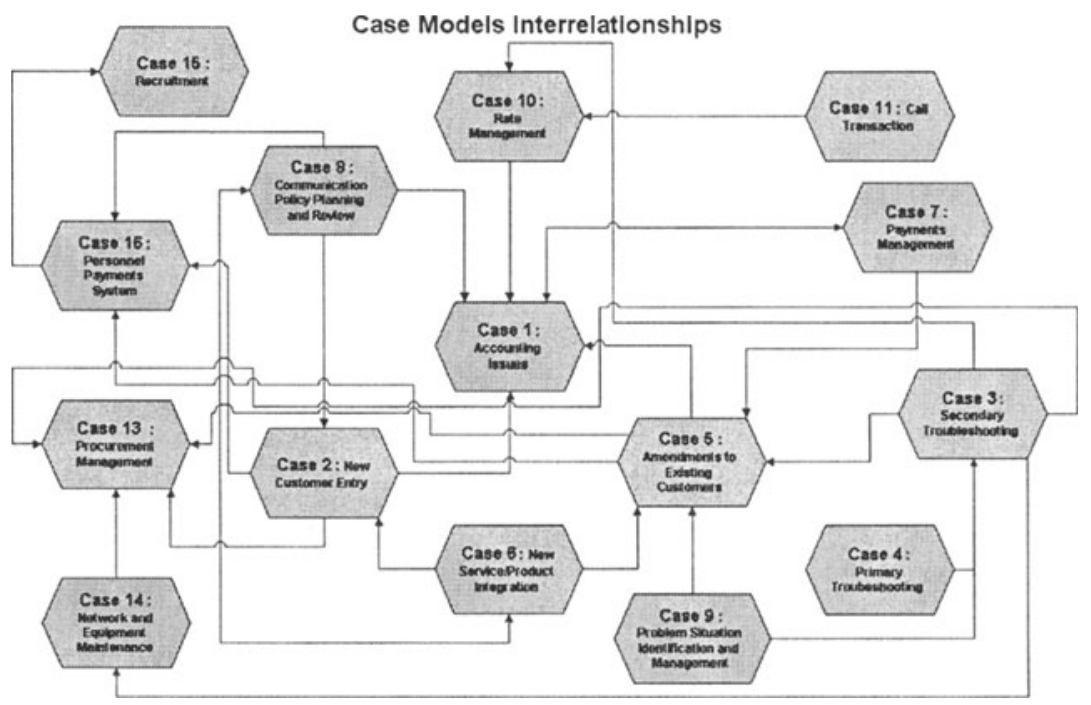

Figure 3: Case Models Interrelationships.

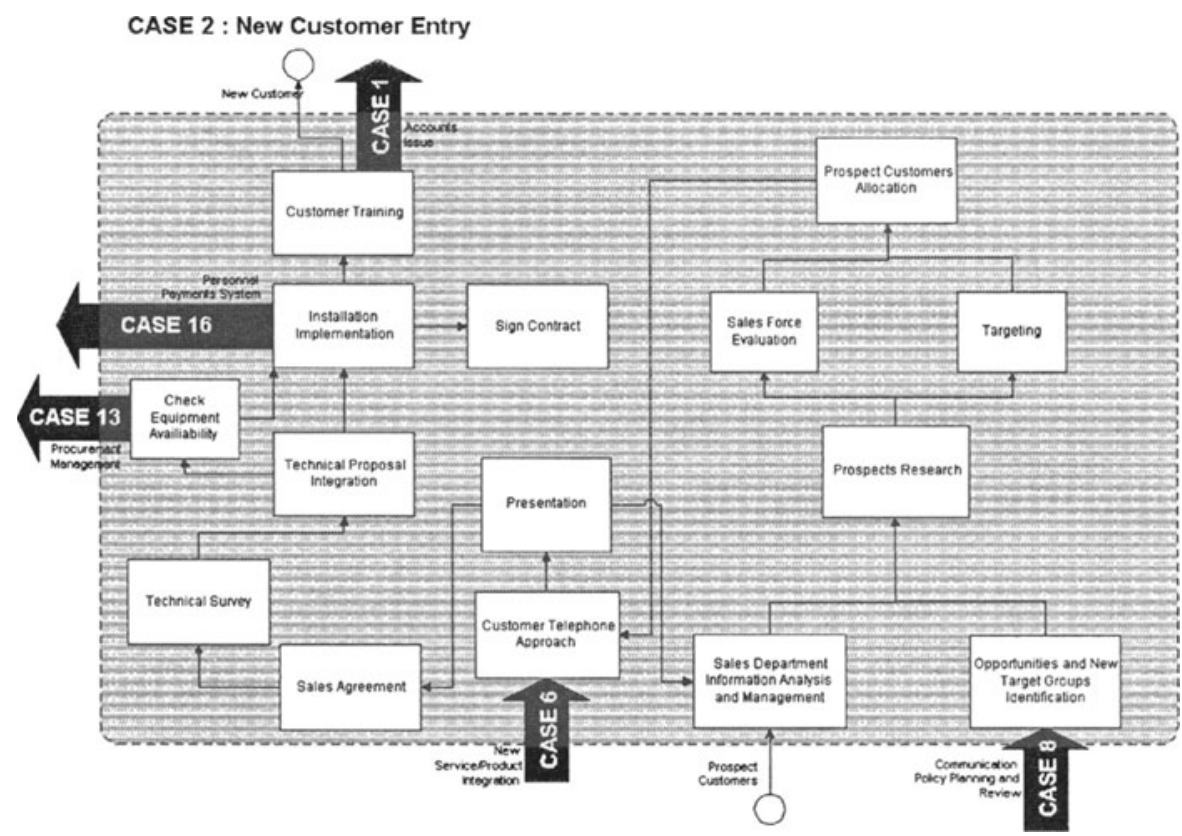

Figure 4: Business Process Analysis Example.

The depiction of processes is implemented using Analytical flow charts, in order to account for the temporal sequence of the processes. The temporal sequence is confined to the order of the particular processes and is 
defined by directed arrows. One such process, specifically, the "Rerouting Decision Making Process" is depicted in Figure 5.

\subsubsection{Rerouting Decision Making Process}

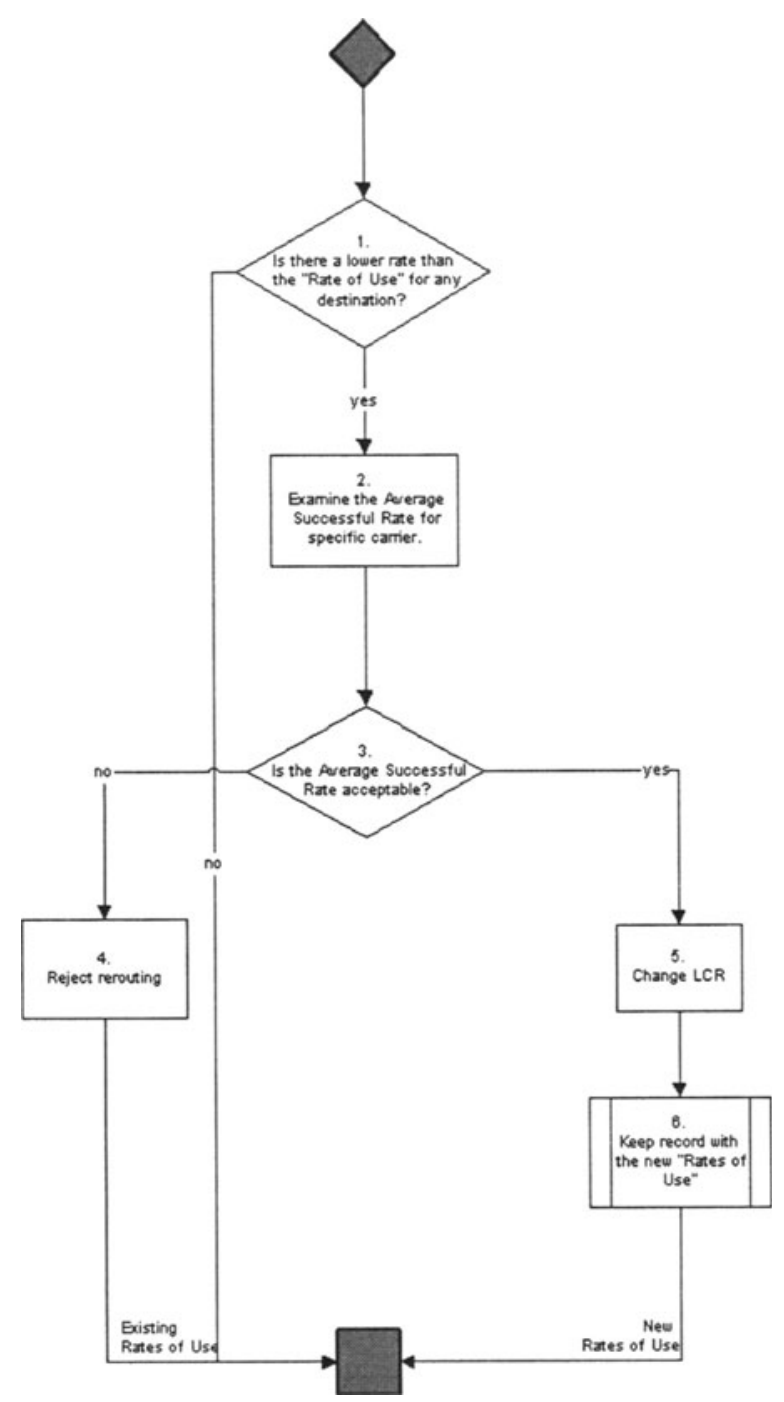

Figure 5: Sample Process.

\section{CONCLUSIONS}

The present work has examined organizational methodologies for an entrant small-size telecom company, which would attempt to meet the above 
criteria. The study introduced the concept of process modeling in a general business environment and analyzed the way in which a telecom company should be organized and structured. The study applied the theoretical approaches to an actual telecom entrant. A SWOT (Strengths-WeaknessesOpportunities-Threats) analysis initially was used to define the operational environment of the corporation. Case models were created as interim stages for the support of the interactions between the business entities. The business processes were then identified, analyzed and evaluated, and typical process flowcharts were generated and documented.

Several interesting conclusions result from this work. First, it is shown that for the successful representation of a company's operation, several software tools for process modelling and documentation are needed. It is recognized that, especially in the telecom market, today's corporations need to adopt a more flexible, process-oriented business structure. The analysis and modelling of business processes facilitates the identification and assignment of roles in an organization chart and enables a company to provide customized, client-oriented services through efficient processes, thus remaining effective and profitable in a competitive, rapidly-changing, disintermediative, digital techno-economic environment.

\section{BIBLIOGRAPHY}

[1] Armistead, C. and Rowland, P. Managing Business Processes: BPR and Beyond. Wiley, 1996.

[2] Espejo, R. et al. Organizational Transformation and Learning: A Cybernetic Approach to Management. Wiley, 1996.

[3] Johnson, Dr. and Edosomwan, A. Organizational Transformation and Process Reengineering. Kogan Page, 1996.

[4] Scheer, A.-W. Business Process Engineering, Reference Models for Industrial Enterprise. Springer-Verlaag, $2^{\text {nd }}$ Edition, 1994.

[5] Scheer, A.-W. ARIS-Business Process Frameworks. Springer-Verlaag, $3^{\text {rd }}$ Edition, 1999.

[6] Scheer, A.-W. ARIS-Business Process Modelling. Springer-Verlaag, $3^{\text {rd }}$ Edition, 1999. 